\title{
Increased Levels of Anxiety Among Medical and Non-Medical University Students During the COVID-19 Pandemic in the United Arab Emirates
}

This article was published in the following Dove Press journal: Risk Management and Healthcare Policy

\author{
Basema Saddik (iD) ${ }^{1,2}$ \\ Amal Hussein' \\ Fatemeh Saheb Sharif-Askari ${ }^{2}{ }^{2}$ \\ Waad Kheder ${ }^{3}$ \\ Mohamad-Hani Temsah (D) 4 \\ Rim Adnan Koutaich $\left.{ }^{5}\right)^{5}$ \\ Enad Sami Haddad (D) ${ }^{5}$ \\ Nora Marwan Al-Roub ${ }^{5}$ \\ Fatema Adel Marhoon ${ }^{5}$ \\ Qutayba Hamid ${ }^{2,5}$ \\ Rabih Halwani ${ }^{2,5}$ \\ 'Department of Family and Community \\ Medicine, College of Medicine, University \\ of Sharjah, Sharjah, United Arab Emirates; \\ ${ }^{2}$ Sharjah Institute of Medical Research, \\ College of Medicine, University of \\ Sharjah, Sharjah, United Arab Emirates; \\ ${ }^{3}$ College of Dental Medicine, University \\ of Sharjah, Sharjah, United Arab Emirates; \\ ${ }^{4}$ Prince Abdullah Ben Khaled Celiac \\ Disease Research Chair, Department of \\ Pediatrics, Faculty of Medicine, King Saud \\ University, Saudi Arabia; ${ }^{5}$ Department of \\ Clinical Sciences, College of Medicine, \\ University of Sharjah, Sharjah, United \\ Arab Emirates
}

Introduction: The COVID-19 pandemic is likely to increase anxiety levels within the community and in particular medical students who are already considered psychologically vulnerable groups. Since the COVID-19 outbreak, no study has yet estimated the effect of this pandemic on university students in the UAE or its impact on the psychological wellbeing of medical students.

Methods and Materials: We surveyed 1485 medical (comprising medical and dental) and non-medical university students across 4 emirates within the UAE. We used an online platform to assess knowledge, sources of information, changes in hygienic behavior, perceptions of fear and worry and anxiety levels using the generalized anxiety disorder 7 (GAD-7) scale. The GAD-7 score was measured at three time points: during hospital visits for medical/dental students, before the introduction of online learning and after online learning for all students.

Results: The majority of students demonstrated high levels of knowledge and utilized reliable sources of information. Non-medical students exercised higher compliance with social restrictions, while medical students practiced better hand hygiene. Almost half of students reported anxiety levels ranging from mild to severe with females reporting higher anxiety scores during hospital visits $(\mathrm{OR}=2.02,95 \% \mathrm{CI}, 1.41$ to 2.91$)$ and medical students reporting lower anxiety levels in comparison to dental students $(\mathrm{OR}=0.61,95 \% \mathrm{CI}, 0.45$ to 0.84). Medical students reported higher levels of anxiety during their clinical rotations which decreased with the introduction of online learning, yet, non-medical students' anxiety levels increased with online learning.

Conclusion: This study provides important information on the initial response and anxiety levels in university students across the UAE during the COVID-19 pandemic. The findings from our study can be used to support the development of effective screening strategies and interventions to build psychological resilience among university students during the COVID19 pandemic or any other public health emergencies in the future.

Keywords: COVID-19, anxiety, medical students, GAD-7, United Arab Emirates

\section{Introduction}

Human coronaviruses ( $\mathrm{HCoVs}$ ) have long been considered inconsequential pathogens, causing the common cold in otherwise healthy people. ${ }^{1}$ However, in the $21 \mathrm{st}$ century, two highly pathogenic $\mathrm{HCoVs}$ - severe acute respiratory syndrome coronavirus (SARS-CoV) and Middle East respiratory syndrome coronavirus (MERS-CoV) - emerged from animal reservoirs to cause global epidemics with alarming morbidity and mortality in 2003 and 2012, respectively. In December 2019, yet another
Correspondence: Rabih Halwani Email rhalwani@sharjah.ac.ae
Risk Management and Healthcare Policy 2020:I3 2395-2406 
pathogenic $\mathrm{HCoV}$, SARS-CoV-2, was recognized in Wuhan, China, causing Coronavirus disease (COVID-19) ${ }^{1}$ and raising intense attention not only within China but internationally. ${ }^{1}$

Since its first detection, COVID-19 has become a major health problem and the global outbreak was declared a pandemic by the World Health Organization (WHO) on March 11th, 2020. ${ }^{2}$ To date, more than 31 million laboratory-confirmed cases have been reported worldwide with $\sim 966,000$ deaths in 184 countries, bringing the crude case fatality rate (CFR) of COVID-19 to $\sim 3.1 \%{ }^{3}$ The first confirmed case of COVID-19 in the United Arab Emirates (UAE) was reported on 29 January 2020. The UAE had 85,595 confirmed cases (10,104 active; 75,086 recovered; 405 deaths; CFR $\sim 0.5 \%$, as of 21 st September 2020). ${ }^{4}$

The COVID-19 pandemic and the uncertainty surrounding its transmission has caused considerable anxiety and fear around the world. ${ }^{5-8}$ Additionally, social distancing, quarantine and lockdown measures imposed by governments to control the spread of the virus have caused disruptions to businesses, employment and education. These disruptions have had multifaceted implications with a sharp rise of mental health problems, including anxiety, depression, stress, sleep disorders and substance use, particularly amongst university students. $^{9-13}$ Specifically, medical and dental students are psychologically vulnerable groups during these unprecedented times, due to the already highly competitive nature of their training, academic pressure, exposure to patients in clinical settings, financial constraints and lack of sleep; which may already contribute to psychological problems associated with stress and anxiety. ${ }^{14-17}$ Additionally, during disease outbreaks, healthcare students are perceived to be at higher risk of infection because of their increased risk of exposure to the virus during clinical training. ${ }^{18,19}$ This has led to higher reports of anxiety among medical students than non-medical students during previous disease outbreaks, ${ }^{20,21}$ particularly due to their fear of transmitting the virus back to their family and loved ones. ${ }^{22}$

In March 2020, all schools and university programs across the UAE transitioned to online learning including clinical training and clerkships for both medical and dental students. The psychological impact of this transition for university students overall and the disruption it may have caused have yet to be investigated in the UAE particularly for clinical training of medical students. Since the COVID-19 outbreak, no study has yet estimated the effect of this pandemic on university students and its impact on the psychological well-being of students. In this study, we aim to assess university student's psychological distress and concerns during the recent COVID-19 pandemic, their degree of perceived information about the disease, and their overall attitude, practices and behaviors during the outbreak. We also evaluate students' perceptions on the precautionary measures in place and the effectiveness of educational strategies such as the use of online learning for reducing anxiety levels in the United Arab Emirates. This makes our study the first in the UAE to discuss this aspect in the current pandemic.

\section{Methods and Materials Design and Participants}

A cross-sectional study design was used to conduct this research during the COVID-19 outbreak in March 2020. Data were collected between March 11 and March 21. University students from medical, dental and nonmedical colleges across the UAE were invited to participate in the research through an online web survey hosted on the platform survey monkey (https://www.surveymon key.com/r/VSYTNQ9) ${ }^{23}$ (S1). All students enrolled in the medical and dental colleges at the University of Sharjah received the survey link through their university emails using convenience sampling. Students from other universities received the survey link through WhatsApp and other social media platforms using snowball sampling where acquaintances sent the link to each other. Through the survey link, the first page explained the research objectives and assured participants anonymity and confidentiality. Students' acceptance indicated their consent to participate in the study. The study was approved by the University of Sharjah Research Ethics Committee (REC20-03-03-02) prior to participant recruitments.

\section{Sample Size}

In this study, we hypothesized that the prevalence of moderate/severe anxiety would be higher in non-medical rather than in the medical students. A study conducted in Saudi Arabia during the MERS-COV outbreak found that $23 \%$ of medical students reported moderate/severe anxiety levels. ${ }^{20}$ Using this proportion, a confidence level of $95 \%$, margin of error of $5 \%$, and study power of $80 \%$, in order to detect a difference of $10 \%$ in the prevalence of moderate/severe anxiety between medical and non-medical students, we calculated the minimum required sample size to be 311 in each group. To account for non-response, the 
sample size was increased by $20 \%$, making the minimum sample size required for this study 373 students for each of the medical and non-medical groups.

\section{Data Collection}

A questionnaire comprising 18 items was used in this study. A modified version of a questionnaire measuring medical student anxiety previously used in Saudi Arabia during the MERS-CoV outbreak was used, after the author's permission. ${ }^{20,22}$ The questions were divided into seven domains comprising 1) demographic questions, 2) change in hygienic behavior, with responses measured on a 4 point Likert scale ranging from very much has changed to no change at all, 3) level of knowledge on statements related to COVID-19 such as transmission, treatment, prognosis and prevention measured by true/false/do not know responses, 4) perceptions of worry and fear associated with COVID-19 measured on a 5-point Likert scale, ranging from very worried to not worried at all, and their opinion about the public fear associated with COVID-19 being justifiable or dysfunctional, measured on a 5-point Likert scale, ranging from strongly agree to strongly disagree, 5) perception of receiving enough information on COVID-19 on a 5-point Likert scale, ranging from strongly agree to strongly disagree, 6) the sources of information students resorted to for information on COVID-19 and 7) Measurement of anxiety levels.

In order to measure anxiety levels, we used the generalized anxiety disorder scale (GAD-7) ${ }^{24}$ which is a 7-item questionnaire asking participants how often they were bothered by each symptom such as feeling nervous, trouble relaxing, irritable and afraid that something awful might happen during the last 2 weeks. Response options were "not at all," "several days," "more than half the days," and "nearly every day," scored as $0,1,2$, and 3, respectively. A score of 10 or greater represents a reasonable cut point for identifying cases of anxiety with a sensitivity of $89 \%$ and specificity of $82 \%$, internal consistency (Cronbach $\alpha=0.92$ ) and Test-retest reliability (intraclass correlation $=0.83$ ). ${ }^{24}$ The GAD-7 has also been identified as a screener for panic disorder, social phobia and PTSD (with a cutoff score of 8 sensitivity $77 \%$ and specificity $82 \%) .{ }^{25}$ In our study, the GAD-7 score was totaled for each student and classified into cut-off points of (0-4 minimal, 5-9 mild, 10-14 moderate and 15-21 severe) levels of anxiety as outlined by Spitzer ${ }^{24}$. Levels of anxiety were measured using the GAD-7 scale at three different time points. In addition to calculating the mean
GAD-7 score, scores were classified into 3 categories (minimal, mild and moderate/severe anxiety).

During the launch of data collection for this study, the UAE Ministry of Education declared university campuses to move to online learning to prevent the spread of COVID-19 and classes moved online on March 8. In order to determine levels of anxiety of students during the transition to online learning, medical and dental students were asked to respond to the GAD-7 questions reflecting back to the time they were attending hospital visits within the previous two weeks. This was to assess whether higher levels of anxiety were associated with hospital visits. All students were asked to respond to the GAD-7 questions reflecting on "before the introduction of online learning", and "after the introduction of online learning".

The final version of the questionnaire was piloted to ensure clarity and consistency between survey items. To ensure face and content validity of the survey instrument, the survey was sent to a group of 9 experts which consisted of students, tutors, faculty and a psychiatrist who reviewed the survey for content accuracy, clarity and comprehensiveness and whether the survey met its objectives. As a result, the phrasing and response items of some questions were modified, and format was edited for clarity and comprehensibility. The final version of the questionnaire can be found as supplementary material.

\section{Statistical Analysis}

Descriptive statistics, including means, medians, frequencies and percentages were used to summarize data and to illustrate the demographic and other selected characteristics of students. Normality of data was tested visually using the Q-Q plots and statistically using the Kolmogorov-Smirnov test. Bivariate analysis using Chisquare $\left(\chi^{2}\right)$ and Mann-Whitney $U$-tests explored the associations between student demographic characteristics and anxiety levels. Spearman correlation coefficient, r, was used to evaluate the association between knowledge score and GAD-7 score. Statistically significant factors in the bivariate analysis were included in the multivariate ordinal logistic regression analyses to determine if they predicted student anxiety levels. The estimates of the strengths of associations were demonstrated by the odds ratio (OR) with a $95 \%$ confidence interval (CI). A twotailed $p<0.05$ was considered statistically significant. Data were analyzed using IBM SPSS Version 25.0. ${ }^{26}$ 


\section{Results}

In total, 1484 students responded to the questionnaire and data were analyzed for 1385 completed surveys (completion rate $93.3 \%$ ) from 4 different emirates across the UAE. The mean age of students was 20 years and most participants were females (72\%). Almost three quarter of the students were studying medicine or dental medicine and from those students, a third (35\%) were in their clinical years of study and were completing clinical ward rotations. Of these students, $12 \%$ reported being in contact with an infected or suspected case of COVID-19. Demographic characteristics are shown in Table 1.

\section{Hygienic Practices}

The majority of students reported increased hand hygiene $(85 \%)$, increased use of hand sanitizer (85.5\%), avoiding people with flu-like symptoms $(80 \%)$ and decreased visits to crowded places $(77 \%)$. A lower proportion of students reported wearing gloves and masks (58\%), decreased social visits $(62 \%)$ or decreased hand shaking $(58 \%)$. We combined medical and dental students together as the medical group, and all other specialties made up the nonmedical group. When medical and non-medical students were compared, more non-medical students avoided being in contact with people with flu-like symptoms, had decreased social visits, decreased visits to crowded places and use of public facilities as displayed in Figure 1.

\section{Knowledge and Perception of Information on COVID-19}

Most students agreed they had heard enough information on the symptoms, prognosis, transmission and prevention of COVID-19. When we compared medical and nonmedical students, significantly more medical students reported having heard sufficient information on COVID19 prognosis, $\chi^{2}(2, \mathrm{~N}=1385)=55.20, \mathrm{p}<0.001$ and COVID-19 transmission, $\chi^{2}(2, \mathrm{~N}=1385)=17.88$, $\mathrm{p}<0.001$ (Table 2). Overall, students had good knowledge of COVID-19 with over $70 \%$ of students answering correctly on the COVID-19 knowledge questions with a median score of 5 (from a maximum score of 7). When we compared participants' knowledge scores by field of study, gender and contact with COVID-19, we found that medical students, females and students who had been in contact with COVID-19 cases had significantly higher knowledge scores than their counterparts, as shown in Table 2 .
Table I Demographic Characteristics of Students $(\mathrm{N}=1385)$

\begin{tabular}{|c|c|c|c|}
\hline & & $\begin{array}{l}\text { Frequency } \\
\text { (n) }\end{array}$ & $\begin{array}{l}\text { Percentage } \\
\text { (\%) }\end{array}$ \\
\hline Age (years) [mean $\pm \mathrm{SD}]$ & $\begin{array}{l}20.5 \\
\pm \\
2.3\end{array}$ & & \\
\hline $\begin{array}{l}\text { Gender } \\
\text { Female } \\
\text { Male }\end{array}$ & & $\begin{array}{l}994 \\
391\end{array}$ & $\begin{array}{l}71.8 \\
28.2\end{array}$ \\
\hline $\begin{array}{l}\text { Emirate } \\
\text { Sharjah } \\
\text { Dubai } \\
\text { Ajman } \\
\text { Al-Ain/RAK }\end{array}$ & & $\begin{array}{l}814 \\
107 \\
446 \\
18\end{array}$ & $\begin{array}{l}58.8 \\
7.7 \\
32.2 \\
1.3\end{array}$ \\
\hline $\begin{array}{l}\text { Field of study } \\
\text { Medical } \\
\text { Dental } \\
\text { Non-medical }\end{array}$ & & $\begin{array}{l}719 \\
323 \\
343\end{array}$ & $\begin{array}{l}51.9 \\
23.3 \\
24.8\end{array}$ \\
\hline $\begin{array}{l}\text { Phase of study for medical/ } \\
\text { dental students } \\
\text { Pre-Clinical } \\
\text { Clinical }\end{array}$ & & $\begin{array}{l}685 \\
361\end{array}$ & $\begin{array}{l}65.5 \\
34.5\end{array}$ \\
\hline $\begin{array}{l}\text { Current ward rotation for } \\
\text { clinical students* } \\
\text { Not in a rotation } \\
\text { High risk rotation } \\
\text { Low risk rotation }\end{array}$ & & $\begin{array}{l}33 \\
25 \\
280\end{array}$ & $\begin{array}{l}9.8 \\
7.4 \\
82.8\end{array}$ \\
\hline $\begin{array}{l}\text { Contact with suspected } \\
\text { COVID-19 patient } \\
\text { Yes } \\
\text { No }\end{array}$ & & $\begin{array}{l}152 \\
1109\end{array}$ & $\begin{array}{l}12.1 \\
87.9\end{array}$ \\
\hline $\begin{array}{l}\text { GAD-7 Score Median } \\
\text { [IQR] } \\
\text { During hospital visits } \\
\text { (medical/dental students) } \\
\text { Minimal } \\
\text { Mild } \\
\text { Moderate } \\
\text { Severe }\end{array}$ & $4[8]$ & $\begin{array}{l}368 \\
166 \\
82 \\
77\end{array}$ & $\begin{array}{l}53.1 \\
24.0 \\
11.8 \\
11.1\end{array}$ \\
\hline $\begin{array}{l}\text { Before online learning (all } \\
\text { students) } \\
\text { Minimal } \\
\text { Mild } \\
\text { Moderate } \\
\text { Severe }\end{array}$ & 4 [9] & $\begin{array}{l}570 \\
274 \\
116 \\
130\end{array}$ & $\begin{array}{l}52.3 \\
25.1 \\
10.6 \\
11.9\end{array}$ \\
\hline $\begin{array}{l}\text { After online learning (all } \\
\text { students) } \\
\text { Minimal }\end{array}$ & $3[8]$ & 620 & 56.9 \\
\hline
\end{tabular}

(Continued) 
Table I (Continued).

\begin{tabular}{|l|l|l|l|}
\hline & & $\begin{array}{l}\text { Frequency } \\
\text { (n) }\end{array}$ & $\begin{array}{l}\text { Percentage } \\
\text { (\%) }\end{array}$ \\
\hline Mild & & 254 & 23.3 \\
Moderate & & 105 & 9.6 \\
Severe & 111 & 10.2 \\
Knowledge Score Median & $5[1]$ & & \\
[IQR] & & & \\
\hline
\end{tabular}

Notes: * High-risk rotation includes Intensive Care Unit, Emergency Rooms, Operating Rooms, Isolation wards.

\section{Student Sources of Information on COVID-19}

Students' main sources of information were official websites, press releases from the Ministry of Health $(\mathrm{MOH})$ and social media. A statistically significant higher percentage of medical students reported using the WHO website $(52 \%) \quad(p \leq 0.001), \quad \mathrm{MOH} \quad(50 \%) \quad(p=0.001)$, hospital announcements $(27 \%)(p \leq 0.001)$ and social media $(54 \%)$ $(p=0.010)$ to retrieve their information as displayed in Figure 2.

\section{Levels of Anxiety and Worry Among Students}

The median GAD-7 scores for medical students during their hospital visits, for all students before introduction of online learning and after online learning were 4, 4 and 3 , respectively. When we compared GAD-7 scores for medical students by gender, specialty (dental or medical), contact with COVID-19, rotation type and clinical phase of study, we found that females, dental medicine students, students who had been in contact with COVID-19 patients and students in their clinical phase of study reported statistically significant higher anxiety levels. More students $(27 \%)$ in low-risk ward rotations reported mild anxiety while the majority of students who reported moderate to severe levels of anxiety (26\%) were attending high-risk ward rotations $\chi^{2}(3, \mathrm{~N}=1385)=10.92, \mathrm{p}=0.027$ (Table 3$)$.

Overall, females reported higher levels of anxiety in GAD-7, both before and after online learning. The medical students reported higher levels of anxiety before online learning in comparison to non-medical students (Table 3).

Interestingly, anxiety levels significantly decreased for females and for medical students after switching to the online learning, with $\chi^{2}(2, \mathrm{~N}=1385)=12.22, \mathrm{p}<0.001$ and $\chi^{2}(2, \mathrm{~N}=1385)=20.99, \mathrm{p}<0.001$, respectively, with a higher percentage of decreased anxiety among dental students; whilst non-medical students reported higher levels of anxiety after the introduction of online learning $\left(\chi^{2}(4, \mathrm{~N}=1385)=36.38, \mathrm{p}<0.001\right)$ (Table 3$)$.

The majority of students $(73 \%)$ reported being worried about transmitting COVID-19 to one of their family members or friends, while $65 \%$ of them were worried about catching the

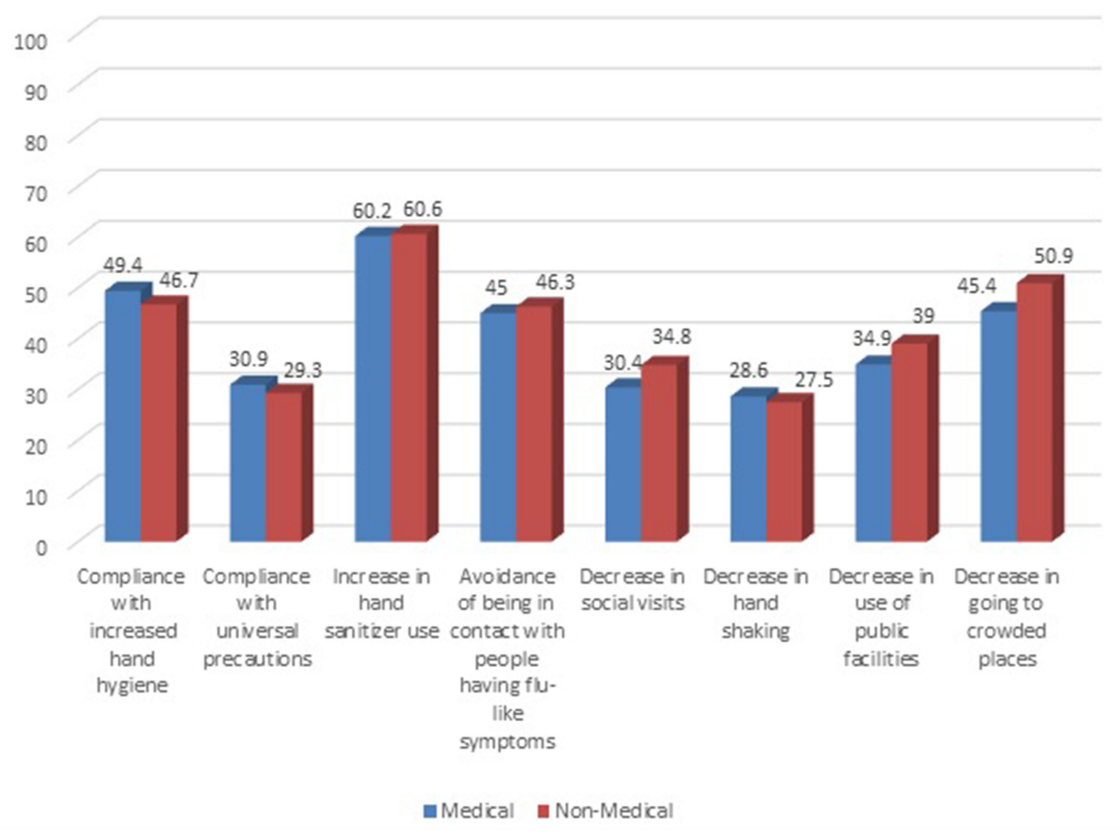

Figure I Proportion of medical and non-medical students reporting "very much change" in hygienic practices related to COVID-19 outbreak. 
Table 2 Perception of Sufficient COVID-I 9 Information by Field of Study and Knowledge of COVID-19 by Demographic Factors

\begin{tabular}{|c|c|c|c|c|c|c|c|c|}
\hline \multicolumn{9}{|c|}{ I believe I Have Heard Sufficient Information About COVID- I9, n (\%) } \\
\hline & & \multicolumn{2}{|c|}{ Strongly Disagree/Disagree } & Neutral & \multicolumn{2}{|c|}{ Agree/Strongly Agree } & Chi-Square* & $P$-value \\
\hline Symptoms & Medical & \multirow{2}{*}{\multicolumn{2}{|c|}{$\begin{array}{l}94(11.2) \\
16(7.8)\end{array}$}} & $87(10.3)$ & \multirow{2}{*}{\multicolumn{2}{|c|}{$\begin{array}{l}660(78.5) \\
162(79.0)\end{array}$}} & 2.99 & 0.224 \\
\hline & Non-medical & & & $27(13.2)$ & & & & \\
\hline Prognosis & Medical & \multirow{2}{*}{\multicolumn{2}{|c|}{$\begin{array}{l}159(18.9) \\
33(16.1)\end{array}$}} & $145(17.2)$ & \multirow{2}{*}{\multicolumn{2}{|c|}{$\begin{array}{l}537(63.9) \\
88(42.9)\end{array}$}} & 55.20 & $<0.0001$ \\
\hline & Non-medical & & & $84(4 I .0)$ & & & & \\
\hline Treatment & Medical & \multirow{2}{*}{\multicolumn{2}{|c|}{$\begin{array}{l}305(36.3) \\
88(42.9)\end{array}$}} & $212(25.2)$ & \multirow{2}{*}{\multicolumn{2}{|c|}{$\begin{array}{l}324(38.5) \\
63(30.7)\end{array}$}} & 4.74 & 0.094 \\
\hline & Non-medical & & & $54(26.3)$ & & & & \\
\hline Transmission & Medical & \multirow{2}{*}{\multicolumn{2}{|c|}{$\begin{array}{l}56(6.7) \\
24(11.7)\end{array}$}} & $60(7.1)$ & \multirow{2}{*}{\multicolumn{2}{|c|}{$\begin{array}{l}725(86.2) \\
152(74.1)\end{array}$}} & 17.88 & $<0.0001$ \\
\hline & Non-medical & & & $29(14.1)$ & & & & \\
\hline Prevention & Medical & \multirow{2}{*}{\multicolumn{2}{|c|}{$\begin{array}{l}37(4.4) \\
13(6.3)\end{array}$}} & $55(6.5)$ & \multirow{2}{*}{\multicolumn{2}{|c|}{$\begin{array}{l}749(89.1) \\
176(85.9)\end{array}$}} & $\mathrm{I} .88$ & 0.391 \\
\hline & Non-medical & & & $16(7.8)$ & & & & \\
\hline \multicolumn{9}{|c|}{ Knowledge score by field of study, gender and contact with COVID-19 patient } \\
\hline \multicolumn{3}{|l|}{ (Maximum possible $0-7$ ) } & \multicolumn{3}{|c|}{ Median Knowledge Score } & \multicolumn{2}{|c|}{ Mann-Whitney U-test } & $P$-value \\
\hline Field of Study & \multicolumn{2}{|l|}{$\begin{array}{l}\text { Medical } \\
\text { Non-Medical }\end{array}$} & \multicolumn{3}{|l|}{$\begin{array}{l}6 \\
5\end{array}$} & \multicolumn{2}{|l|}{$75,060.5$} & $<0.0001$ \\
\hline Gender & \multirow{2}{*}{\multicolumn{2}{|c|}{$\begin{array}{l}\text { Female } \\
\text { Male }\end{array}$}} & \multirow{2}{*}{\multicolumn{3}{|c|}{$\begin{array}{l}6 \\
5\end{array}$}} & \multirow{2}{*}{\multicolumn{2}{|c|}{$|27,28|$}} & $<0.0001$ \\
\hline & & & & & & & & \\
\hline \multirow[t]{2}{*}{ Contact with COVID-19 } & \multicolumn{2}{|l|}{ Yes } & \multicolumn{3}{|l|}{6} & \multicolumn{2}{|l|}{68,041} & 0.011 \\
\hline & \multicolumn{2}{|l|}{ No } & \multicolumn{3}{|l|}{5} & & & \\
\hline
\end{tabular}

Note: $*$ df $($ Degrees of freedom $)=2$.

virus themselves. Almost half (49\%) of medical students reported being worried about transmitting COVID-19 to others and were less likely (29\%) to worry about catching it themselves, in comparison to non-medical students (35\%), although the differences were not statistically significant $\left(\chi^{2}(2, \mathrm{~N}=1385)=2.85, \mathrm{p}=0.24\right)$. Most students $(61 \%)$ agreed that the public fear was justifiable and (46\%) of students did not perceive it as dysfunctional.

A cumulative odds ordinal logistic regression with proportional odds was performed to determine predictors

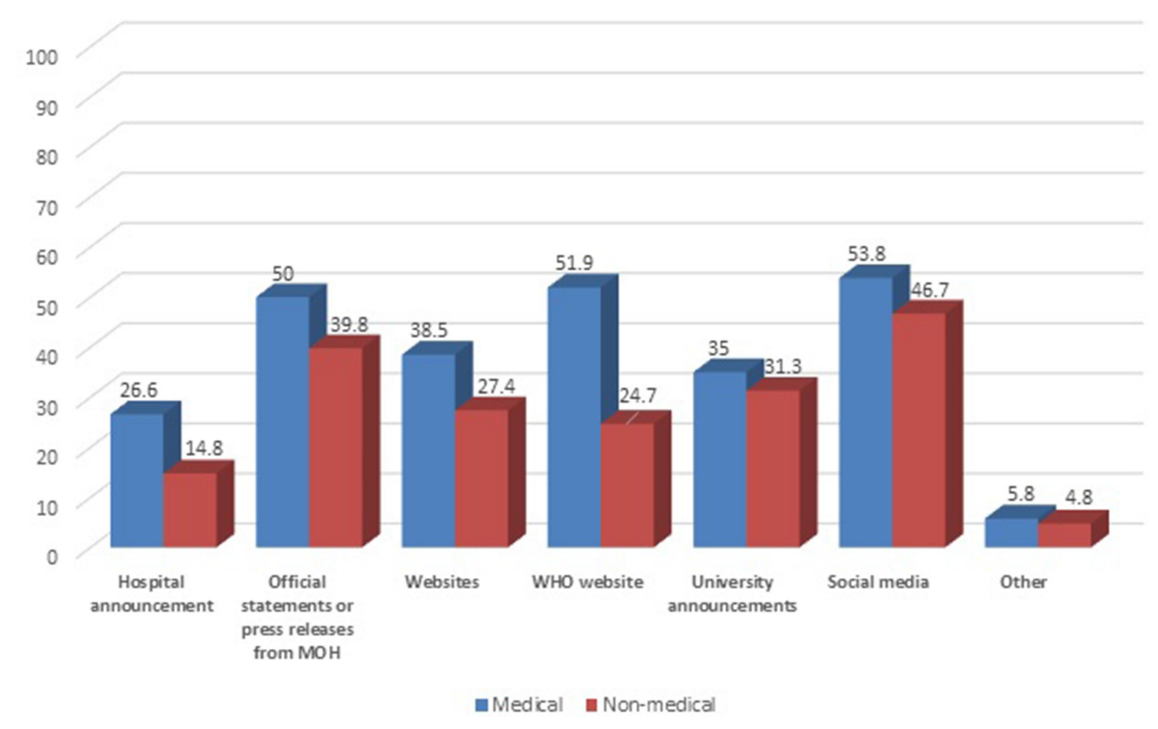

Figure 2 Sources of information for COVID-19 for medical and non-medical students. 
Table 3 Generalized Anxiety Disorder-7 (GAD-7) Scores (0-2I) During Hospital Visits, Before and After Online Learning by Sociodemographic Factors

\begin{tabular}{|c|c|c|c|c|c|c|c|}
\hline \multicolumn{8}{|c|}{ GAD-7 Score During Hospital Visits (Medical/Dental Students) n (\%) } \\
\hline & & Minimal (0-4) & Mild (5-9) & Moderate/Severe $(10-2 I)$ & Chi-Square & df & $P$-value* \\
\hline \multirow[t]{2}{*}{ Gender } & Female & $296(50.2)$ & $149(25.3)$ & $145(24.6)$ & 19.584 & 2 & $\leq 0.0001$ \\
\hline & Male & $135(67.5)$ & $39(19.5)$ & $26(13)$ & & & \\
\hline \multirow[t]{2}{*}{ Specialty } & Medical & $243(58.1)$ & $95(22.7)$ & $80(19.2)$ & 7.535 & 2 & 0.023 \\
\hline & Dental & $116(47.5)$ & $64(26.2)$ & $64(26.2)$ & & & \\
\hline \multirow[t]{2}{*}{ Contact with COVID-19 } & No & $324(55.9)$ & $140(24.1)$ & $116(20)$ & 18.586 & 2 & $\leq 0.0001$ \\
\hline & Yes & $44(38.9)$ & $26(23)$ & $43(38.1)$ & & & \\
\hline \multirow[t]{3}{*}{ Rotation type } & No Rotation & $199(58.4)$ & $78(22.9)$ & $64(18.8)$ & 10.92 & 3 & 0.027 \\
\hline & High risk** & $18(66.7)$ & $2(7.4)$ & $7(25.9)$ & & & \\
\hline & Low risk & $142(48.3)$ & $79(26.9)$ & $73(24.8)$ & & & \\
\hline \multirow[t]{2}{*}{ Clinical Phase of study } & Pre-clinical & $211(58.4)$ & $82(22.7)$ & $68(18.8)$ & 6.627 & 2 & 0.043 \\
\hline & Clinical & $148(49.2)$ & $77(25.6)$ & $76(25.2)$ & & & \\
\hline \multicolumn{8}{|c|}{ GAD-7 score before online learning (all students) $n$ (\%) } \\
\hline \multirow[t]{2}{*}{ Gender } & Female & $376(47.8)$ & $216(27.5)$ & $194(24.7)$ & 22.435 & 2 & $\leq 0.0001$ \\
\hline & Male & $194(63.8)$ & $58(19.1)$ & $52(17.1)$ & & & \\
\hline \multirow[t]{2}{*}{ Field of Study } & Medical & $484(55.7)$ & $213(24.5)$ & $172(19.8)$ & 24.793 & 2 & $\leq 0.0001$ \\
\hline & Non-medical & $86(38.9)$ & $61(27.6)$ & $74(33.5)$ & & & \\
\hline \multicolumn{8}{|c|}{ GAD-7 score after online learning (all students) $n$ (\%) } \\
\hline \multirow[t]{2}{*}{ Gender } & Female & $423(53.8)$ & $196(24.9)$ & $167(21.2)$ & 10.788 & 2 & 0.005 \\
\hline & Male & $197(64.8)$ & $58(19.1)$ & $49(16.1)$ & & & \\
\hline \multirow[t]{2}{*}{ Field of Study } & Medical & $546(62.8)$ & $194(22.3)$ & $129(14.8)$ & 81.90 & 2 & $\leq 0.0001$ \\
\hline & Non-medical & $74(33.5)$ & $60(27.1)$ & $87(39.4)$ & & & \\
\hline \multicolumn{8}{|c|}{ Change in GAD-7 scores before and after online learning $n(\%)$} \\
\hline 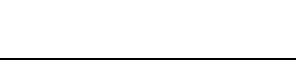 & & Before $>$ After & Before=After & Before $<$ After & & & \\
\hline \multirow{2}{*}{ Gender } & Female & $184(23.4)$ & $479(60.9)$ & $123(15.6)$ & 12.224 & 2 & 0.0002 \\
\hline & Male & $47(15.5)$ & $219(72)$ & $38(12.5)$ & & & \\
\hline \multirow[t]{3}{*}{ Specialty } & Medical & $109(18.2)$ & $405(67.5)$ & $86(14.3)$ & 36.383 & 4 & $\leq 0.0001$ \\
\hline & Dental & $77(28.6)$ & $17 \mid(63.6)$ & $21(7.8)$ & & & \\
\hline & Non-medical & $45(20.4)$ & $122(55.2)$ & $54(24.4)$ & & & \\
\hline \multirow[t]{2}{*}{ Field of Study } & Medical & $186(2 \mid .4)$ & $576(66.3)$ & $107(12.3)$ & 20.994 & 2 & $\leq 0.0001$ \\
\hline & Non-medical & $45(20.4)$ & $122(55.2)$ & $54(24.4)$ & & & \\
\hline \multirow[t]{2}{*}{ Contact with COVID-19 } & No & $200(20.9)$ & $612(64)$ & $144(15.1)$ & 0.722 & 2 & 0.697 \\
\hline & Yes & $31(23.1)$ & $86(64.2)$ & $17(12.7)$ & & & \\
\hline
\end{tabular}

Notes: *Significant at $p<0.05$. ** High-risk rotation includes Intensive Care Unit, Emergency Room, Operating Rooms, Isolation wards.

of anxiety levels during the different time points. The effect of gender, specialty, contact with COVID-19, rotation type, clinical phase of study and knowledge score on the GAD-7 score during hospital visits was modelled. We included knowledge score in the regression model because we found a positive, but weak, correlation between knowledge score and anxiety score using Spearman correlation $\left(\mathrm{r}_{\mathrm{s}}=0.086, \mathrm{p}=0.015\right)$. The odds of females having higher anxiety scores during hospital visits was $2.02(95 \% \mathrm{CI}, 1.41$ to 2.91$)$ times 
that for males, and medical students reported lower anxiety levels in comparison to dental medicine students ( $\mathrm{OR}=0.61,95 \% \mathrm{CI}, 0.45$ to 0.84 ). Furthermore, being in a rotation and demonstrating higher knowledge scores predicted higher GAD-7 scores during hospital visits
(Table 4). Similarly, gender, field of study and knowledge score predicted higher GAD-7 scores before online learning, but only gender and field of study predicted GAD-7 scores after switching to online learning (Table 4).

Table 4 Ordinal Logistic Regression Model for Factors Predicting Anxiety GAD-7 Score

\begin{tabular}{|c|c|c|c|c|c|}
\hline & n (\%) & B & SE(B) & P-value & OR $[95 \% \mathrm{Cl}]$ \\
\hline \multicolumn{6}{|c|}{ GAD-7 Score During Hospital Visits } \\
\hline \multicolumn{6}{|l|}{ Gender } \\
\hline Female & $492(74.3)$ & 0.71 & 0.19 & $\leq 0.001$ & $2.02[|.4|-2.9 \mid]$ \\
\hline Male $^{\mathrm{a}}$ & $170(25.7)$ & - & - & - & 1 \\
\hline Specialty Medical & $418(63.1)$ & -0.49 & 0.16 & 0.002 & $0.61[0.45-0.84]$ \\
\hline Dental $^{\mathrm{a}}$ & $244(36.9)$ & - & - & - & 1 \\
\hline \multicolumn{6}{|c|}{ Contact with COVID-19 } \\
\hline No & $552(83.4)$ & -0.77 & 0.21 & $\leq 0.001$ & $0.46[0.31-0.69]$ \\
\hline Yes $^{\mathrm{a}}$ & $110(16.6)$ & - & - & - & I \\
\hline \multicolumn{6}{|l|}{ In Rotation } \\
\hline No & $34 \mid(5 \mid .5)$ & -0.09 & 0.22 & 0.672 & $0.91[0.60-1.40]$ \\
\hline Yes $^{\mathrm{a}}$ & $321(48.5)$ & - & - & - & I \\
\hline \multicolumn{6}{|l|}{ Clinical phase of study } \\
\hline Pre-clinical & $361(54.5)$ & -0.04 & 0.22 & 0.856 & $0.96[0.62-1.47]$ \\
\hline Clinical $^{\mathrm{a}}$ & $30 I(45.5)$ & - & - & & \\
\hline Knowledge score & 662 & 0.18 & 0.08 & 0.024 & $1.19[1.02-1.40]$ \\
\hline \multicolumn{6}{|c|}{-2Log-Likelihood 424.53 Likelihood Ratio Chi-Square $48.790(\mathrm{df}=6, \mathrm{p}$-value $\leq 0.00 \mathrm{I})$} \\
\hline \multicolumn{6}{|c|}{ GAD-7 score before online learning } \\
\hline \multicolumn{6}{|l|}{ Gender } \\
\hline Female & $786(72.1)$ & 0.61 & 0.14 & $\leq 0.001$ & $1.85[|.4|-2.4 \mid]$ \\
\hline $\mathrm{Male}^{\mathrm{a}}$ & $304(27.9)$ & - & - & - & I \\
\hline \multicolumn{6}{|l|}{ Field of study } \\
\hline Medical & $869(79.7)$ & -0.87 & 0.15 & $\leq 0.001$ & $0.42[0.31-0.56]$ \\
\hline Non-medical ${ }^{a}$ & $221(20.3)$ & - & - & - & 1 \\
\hline Knowledge score & 1090 & 0.18 & 0.06 & 0.002 & $1.20[1.07-1.34]$ \\
\hline \multicolumn{6}{|c|}{-2Log-Likelihood 208.599 Likelihood Ratio Chi-Square 58.130 (df=3, p-value $\leq 0.001)$} \\
\hline \multicolumn{6}{|c|}{ GAD score after online learning } \\
\hline \multicolumn{6}{|l|}{ Gender } \\
\hline Female & $786(72.1)$ & $0.54 I$ & 0.141 & $\leq 0.001$ & $1.72[1.30-2.26]$ \\
\hline Male $^{\mathrm{a}}$ & 304 (27.9) & - & - & - & I \\
\hline \multicolumn{6}{|l|}{ Field of study } \\
\hline Medical & 869 (79.7) & -1.389 & 0.152 & $\leq 0.001$ & $0.25[0.189-0.34]$ \\
\hline Non-medical ${ }^{\mathrm{a}}$ & $221(20.3)$ & - & - & - & 1 \\
\hline Knowledge score & 1090 & 0.090 & 0.058 & 0.122 & $1.10[0.98-1.23]$ \\
\hline
\end{tabular}




\section{Discussion}

This study has revealed that the COVID-19 pandemic has impacted on anxiety levels among university students in the UAE, with almost half of students reporting mild to moderate/severe anxiety levels. The effect of COVID-19 on the global community overall has been considerably significant, causing fear, anxiety and worry, particularly due to uncertainty of the prognosis of the disease, changes in societies lifestyles, lockdown restrictions and educational disruptions. The impact of COVID-19 on university students is particularly burdensome due to the perceived effect of the virus on their studies; and with medical students specifically, it could be due to the interdisciplinary nature of their training and the potential proximity of being exposed to the virus during their clinical studies. ${ }^{27}$

Overall, students in our study demonstrated good knowledge of COVID-19 and reported using reliable sources such as official statements and press releases from the ministry of health and the WHO website. Although the use of these sources was higher among medical students, social media was the main source of information for both medical and nonmedical students. This finding is consistent with the literature and emphasizes the role that social media can play in risk perception and dissemination of reliable information during a pandemic such as COVID-19. ${ }^{28-31}$ However, other studies have also reported that young people tend to obtain a large amount of information from social media which can easily be a trigger and predictor for stress and anxiety. ${ }^{6,32,33}$

Good knowledge may also explain students' compliance with hygienic practices in our study. Most students reported significant change in their hygienic behavior since the COVID19 outbreak, particularly for increased hand hygiene, avoiding crowded places and avoiding being in contact with people with flu-like symptoms. However, even though levels of knowledge were significantly higher among medical students, compliance with hygienic practices was similar for both groups. Furthermore, we found significant positive correlations between changes in hygienic behavior and increased levels of anxiety. More than half of students who reported changed hygienic practices reported higher levels of anxiety, which is consistent with recent literature indicating that people who were more anxious about COVID-19 were also more engaged with regular hand hygiene and social distancing behaviors. ${ }^{34}$

There is increasing evidence that the number of growing cases of COVID-19 globally and within the UAE is causing public worry and concern ${ }^{35,36}$ and in the absence of vaccines and effective treatment, government authorities have introduced rules and restrictions. ${ }^{37}$ Compliance and adherence with these restrictions has been found to differ amongst different age groups and populations, with less acceptance of these restrictions being reported amongst younger age-groups. ${ }^{38}$ However, in our study, the majority of students did not consider the strict measures undertaken by the healthcare authorities as dysfunctional or not required. The students were worried about transmitting COVID-19 to their family members more than they were worried about catching the virus themselves, therefore, indicating a beneficial sense of social responsibility during such infectious disease outbreak. Similar concerns were reported amongst healthcare workers in Hong Kong and Canada during the SARS outbreak. ${ }^{39,40}$

The results from the current study confirm that anxiety levels due to COVID-19 among university students are high, ranging from mild to severe, especially amongst females, which is consistent with previous research. ${ }^{5,6,17,41}$ The majority of students in our study were females, which reflects the gender imbalance in higher education within the UAE. More than two thirds of students reported mild anxiety levels and one third of students reported severe anxiety. What makes this study unique, is that medical and non-medical students were compared, and within the medical student group, we compared medical and dental students. Furthermore, this study was conducted during a critical time at the onset of the pandemic and during the transition to online learning allowing us to assess anxiety levels at three different time points: during hospital visits for medical and dental students, before online learning and after switching to the online learning for all university students. Among the medical/dental group, students in their clinical phase of study who had rotations in high-risk wards or who had been in contact with COVID-19 patients reported significantly higher (moderate to severe) levels of anxiety, demonstrating that high-risk perception of COVID-19 may contribute to higher levels of anxiety. Medical/dental students continued attending the hospitals at the early onset of COVID19 when fear and worry associated with the outbreak would have been at their peak and before lockdown restrictions were in place, which could also explain their high compliance with infection control measures. Medical students' high-risk perceptions associated with attending hospitals during infectious disease outbreaks have been reportedly associated with higher levels of anxiety ${ }^{21,31}$ and higher scores on fear scales and anxiety levels among health care workers. ${ }^{42}$ However, when we compared between medical and dental students, dental students reported higher levels of anxiety and these remained significant with further multivariate analysis. Previous studies have established that medical and dental programs are highly 
competitive, and students generally experience high levels of stress during their training. ${ }^{43,44}$ Yet, dental students suffer from greater levels of perceived stress than medical students due to their role as providers of care and earlier exposure to patients in the dental clinics. ${ }^{45,46}$ Furthermore, dental students are in very close proximity to patients and are dealing directly with patients' dental care during a potentially highly transmissible respiratory virus with increased potential to become infected due to the nature of the field itself. ${ }^{47}$ This may explain the higher levels of anxiety among dental students in our study and the overall decrease in anxiety among all medical students following the introduction of online learning. Psychological support should be tailored to each student's needs and incorporated into the online remote curriculum. Screening university students on a regular basis with tools such as the GAD-7 could help faculty in the early identification of highly anxious students and guide students to receive help from targeted interventions that promote psychological well-being, offer pastoral counselling, mental health support and coping mechanisms. ${ }^{48,49}$

Non-medical students reported higher levels of anxiety before and after online learning in comparison to medical students and whilst medical student anxiety levels decreased following the introduction of online learning, non-medical students' anxiety levels increased. This may be due to several factors, including medical students possibly being more familiar with the use of online learning platforms, being distant from the perceived risk of COVID-19, or due to the variable sources of information about the pandemic among both groups. Knowledge of the virus might reduce students' fears and anxiety while inadequate understanding of COVID-19, its prognosis, transmission and control measures might contribute to negative implications and fear of the unknown ${ }^{40}$ hence explaining the higher anxiety levels in non-medical students in our study.

\section{Limitations}

Despite the findings of this study, we acknowledge that it has several limitations. Firstly, the use of convenience sampling and its descriptive nature through an online survey may not allow the generalization of results especially since anxiety may be due to many other factors other than COVID-19 and which may not be captured through this method. However, considering the need for a rapid method to assess stress and anxiety in a vulnerable population during a rapidly evolving infectious disease outbreak, the use of an online survey serves as a promising method for quick results. ${ }^{50}$ Additionally, responses were collected from four different emirates across the UAE with good response rate allowing for a certain element of representation. Secondly, the nature of self-reported data in the survey may lead to response biases specifically for hygienic practices where students may provide socially desirable responses and self-reported levels of anxiety, stress and worry which may not always be as accurate as being assessed by a mental health professional. However, despite these limitations, this study provides important baseline information which will inform further research and public health interventions in this area.

\section{Conclusion}

To the best of our knowledge, this is the first study to provide important information on the initial response and anxiety levels in university students across the UAE immediately following the period COVID-19 was declared a global pandemic. More than half of university students reported mild to severe anxiety levels with a quarter of students reporting severe anxiety. Specifically, medical students reported higher levels of anxiety during their clinical rotations which decreased with the introduction of online learning, yet, nonmedical student's anxiety levels increased with online learning. The findings from our study can be used at the government and university level to develop effective screening strategies and to formulate interventions that improve mental health of students. Such strategies will reduce unnecessary stress and anxiety among university students, as well as build psychological resilience during the COVID-19 pandemic or any other public health emergencies in the future.

\section{Data Sharing Statement}

The datasets generated during and/or analyzed during the current study are available from the corresponding author on reasonable request.

\section{Ethical Standards}

The authors assert and confirm that all research was performed in accordance with relevant guidelines and regulations of national and institutional committees at the University of Sharjah on human experimentation.

\section{Acknowledgments}

The authors would like to thank all students who participated in the study. 


\section{Author Contributions}

All authors made a significant contribution to the work reported, whether that is in the conception, study design, execution, acquisition of data, analysis and interpretation, or in all these areas; took part in drafting, revising or critically reviewing the article; gave final approval of the version to be published; have agreed on the journal to which the article has been submitted; and agree to be accountable for all aspects of the work.

\section{Funding}

This research has been financially supported by Clinical Epidemiology Research group operational grant (Grant code: 150389); COVID-19 research grant (CoV19-0301) to BS, University of Sharjah, UAE; COVID-19 research grant (CoV19-0307), Seed grant (Grant code: 2001090275); and collaborative research grant (Grant code: 2001090278) to RH, University of Sharjah, UAE; and by Prince Abdullah Ben Khalid Celiac Disease Research Chair, under the Vice Deanship of Research Chairs, King Saud University, Riyadh, Kingdom of Saudi Arabia.

\section{Disclosure}

The authors declare that the research was conducted in the absence of any commercial or financial relationships that could be construed as a potential conflict of interest. The authors report no conflicts of interest for this work.

\section{References}

1. Paules CI, Marston HD, Fauci AS. Coronavirus infections - more than just the common cold. JAMA. 2020;323(8):707. doi:10.1001/ jama.2020.0757

2. WHO. World Health Organisation Director General's opening remarks at the media briefing on COVID-19-11th March 2020 Available from: https://wwwwhoint/dg/speeches/detail/who-directorgeneral-s-opening-remarks-at-the-media-briefing-on-covid-19-11march-2020.

3. World Health Organization. Coronavirus disease (COVID-19) outbreak. Available from: https://covid19whoint/.

4. DOH. Department of Health (UAE). Available from: https://wwwdoh govae/covid-19.

5. Wang C, Pan R, Wan X, et al. Immediate psychological responses and associated factors during the initial stage of the 2019 coronavirus disease (COVID-19) epidemic among the general population in China. Int J Environ Res Public Health. 2020;17(5).

6. Qiu J, Shen B, Zhao M, Wang Z, Xie B, Xu Y. A nationwide survey of psychological distress among Chinese people in the COVID-19 epidemic: implications and policy recommendations. Gen Psychiatr. 2020;33(2):e100213.
7. Wang C, Pan R, Wan X, et al. A longitudinal study on the mental health of general population during the COVID-19 epidemic in China. Brain Behav Immun. 2020;87:40-48. doi:10.1016/j.bbi.2020.04.028

8. Bueno-Notivol J, Gracia-García P, Olaya B, Lasheras I, LópezAntón R, Santabárbara J. Prevalence of depression during the COVID-19 outbreak: a meta-analysis of community-based studies. Int J Clin Health Psychol. 2020. doi:10.1016/j.ijchp.2020.07. 007

9. Gritsenko V, Skugarevsky O, Konstantinov V, et al. COVID-19 fear, stress, anxiety, and substance use among Russian and Belarusian university students. Int J Ment Health Addict. 2020;1-7.

10. Savitsky B, Findling Y, Ereli A, Hendel T. Anxiety and coping strategies among nursing students during the COVID-19 pandemic. Nurse Educ Pract. 2020;46:102809. doi:10.1016/j.nepr.2020.102 809

11. Galea S, Merchant RM, Lurie N. The mental health consequences of COVID-19 and physical distancing: the need for prevention and early intervention. JAMA Intern Med. 2020;180(6):817-818. doi:10.1001/ jamainternmed.2020.1562

12. Islam MA, Barna SD, Raihan H, Khan MNA, Hossain MT, Pakpour AH. Depression and anxiety among university students during the COVID-19 pandemic in Bangladesh: a web-based cross-sectional survey. PLoS One. 2020;15(8):e0238162. doi:10.1371/journal.pone.0238162

13. Sundarasen S, Chinna K, Kamaludin K, et al. Psychological impact of COVID-19 and lockdown among university students in Malaysia: implications and policy recommendations. Int J Environ Res Public Health. 2020;17(17):6206. doi:10.3390/ijerph17176206

14. Al Saadi T, Zaher Addeen S, Turk T, Abbas F, Alkhatib M. Psychological distress among medical students in conflicts: a cross-sectional study from Syria. BMC Med Educ. 2017;17(1):173. doi:10.1186/s12909-017-1012-2

15. Quek TT, Tam WW, Tran BX, et al. The global prevalence of anxiety among medical students: a meta-analysis. Int $J$ Environ Res Public Health. 2019;16(15):2735. doi:10.3390/ijerph16152735

16. Trivate T, Dennis AA, Sholl S, Wilkinson T. Learning and coping through reflection: exploring patient death experiences of medical students. BMC Med Educ. 2019;19(1):451. doi:10.1186/s12909-0191871-9

17. Alahmadi AM. Prevalence of anxiety among college and school students in Saudi Arabia: a systematic review. J Health Inform Dev Ctries. 2019;13(1).

18. Kim S, Kim S. Exploring the determinants of perceived risk of Middle East Respiratory Syndrome (MERS) in Korea. Int J Environ Res Public Health. 2018;15(6).

19. Al Ghobain M, Aldrees T, Alenezi A, et al. Perception and attitude of emergency room resident physicians toward Middle East Respiratory Syndrome Outbreak. Emerg Med Int. 2017;2017:6978256. doi:10.1155/2017/6978256

20. Al-Rabiaah A, Temsah MH, Al-Eyadhy AA, et al. Middle East Respiratory Syndrome-Corona Virus (MERS-CoV) associated stress among medical students at a university teaching hospital in Saudi Arabia. J Infect Public Health. 2020;13(5):687-691. doi:10.1016/j. jiph.2020.01.005

21. Wong TW, Gao Y, Tam WWS. Anxiety among university students during the SARS epidemic in Hong Kong. Stress Health. 2007;23 (1):31-35. doi:10.1002/smi.1116

22. Goulia P, Mantas C, Dimitroula D, Mantis D, Hyphantis T. General hospital staff worries, perceived sufficiency of information and associated psychological distress during the $\mathrm{A} / \mathrm{H} 1 \mathrm{~N} 1$ influenza pandemic. BMC Infect Dis. 2010;10:322. doi:10.1186/1471-2334-10-322

23. SurveyMonkey. San Mateo. CA. Available from: www.surveymon key.com.

24. Spitzer RL, Kroenke K, Williams JB, Lowe B. A brief measure for assessing generalized anxiety disorder: the GAD-7. Arch Intern Med. 2006;166(10):1092-1097. doi:10.1001/archinte.166.10.1092 
25. Kroenke K, Spitzer RL, Williams JB, Monahan PO, Lowe B. Anxiety disorders in primary care: prevalence, impairment, comorbidity, and detection. Ann Intern Med. 2007;146(5):317-325. doi:10.7326/00034819-146-5-200703060-00004

26. IBM Corp. IBM SPSS Statistics for Windows VA. NY: IBM Corp; 2017.

27. Rastegar Kazerooni A, Amini M, Tabari P, Moosavi M. Peer mentoring for medical students during COVID-19 pandemic via a social media platform. Med Edu. 2020;54(8):762-763. doi:10.1111/ medu. 14206

28. Albarrak AI, Mohammed R, Al Elayan A, et al. Middle East Respiratory Syndrome (MERS): comparing the knowledge, attitude and practices of different health care workers. J Infect Public Health. 2019. doi:10.1016/j.jiph.2019.06.029

29. Karasneh R, Al-Azzam S, Muflih S, Soudah O, Hawamdeh S, Khader Y. Media's effect on shaping knowledge, awareness risk perceptions and communication practices of pandemic COVID-19 among pharmacists. Res Social Admin Pharm. 2020. doi:10.1016/j. sapharm.2020.04.027

30. Khan MU, Shah S, Ahmad A, Fatokun O. Knowledge and attitude of healthcare workers about Middle East Respiratory Syndrome in multispecialty hospitals of Qassim, Saudi Arabia. BMC Public Health. 2014;14:1281. doi:10.1186/1471-2458-14-1281

31. Yang S, Cho SI. Middle East respiratory syndrome risk perception among students at a university in South Korea, 2015. Am J Infect Control. 2017;45(6):e53-e60.

32. Chao M, Xue D, Liu T, Yang H, Hall BJ. Media use and acute psychological outcomes during COVID-19 outbreak in China. J Anxiety Disord. 2020;74:102248. doi:10.1016/j.janxdis.2020.102248

33. Mertens G, Gerritsen L, Duijndam S, Salemink E, Engelhard IM. Fear of the coronavirus (COVID-19): predictors in an online study conducted in March 2020. J Anxiety Disord. 2020;74:102258. doi:10.1016/j.janxdis.2020.102258

34. Harper CA, Satchell LP, Fido D, Latzman RD. Functional fear predicts public health compliance in the COVID-19 pandemic. Int $J$ Ment Health Addict. 2020;1-14.

35. Bao Y, Sun Y, Meng S, Shi J, Lu L. 2019-nCoV epidemic: address mental health care to empower society. Lancet (London, England). 2020;395(10224):e37-e38. doi:10.1016/S0140-6736(20)30309-3

36. Rajkumar RP. COVID-19 and mental health: a review of the existing literature. Asian J Psychiatr. 2020;52:102066. doi:10.1016/j. ajp.2020.102066

37. Ryan BJ, Coppola D, Canyon DV, Brickhouse M, Swienton R. COVID-19 community stabilization and sustainability framework: an integration of the Maslow hierarchy of needs and social determinants of health. Disaster Med Public Health Prep. 2020;1-16.
38. Zettler I, Christoph S, Lau L, Robert B. Individual differences in accepting personal restrictions to fight the COVID-19 pandemic: results from a Danish adult sample. PsyArXiv. 2020.

39. Maunder RG, Lancee WJ, Balderson KE, et al. Long-term psychological and occupational effects of providing hospital healthcare during SARS outbreak. Emerg Infect Dis. 2006;12(12):1924-1932. doi:10.3201/eid1212.060584

40. Wong JG, Cheung EP, Cheung V, et al. Psychological responses to the SARS outbreak in healthcare students in Hong Kong. Med Teach. 2004;26(7):657-659. doi:10.1080/01421590400006572

41. Liu N, Zhang F, Wei C, et al. Prevalence and predictors of PTSS during COVID-19 outbreak in China hardest-hit areas: gender differences matter. Psychiatry Res. 2020;287:112921. doi:10.1016/j. psychres.2020.112921

42. Lu W, Wang H, Lin Y, Li L. Psychological status of medical workforce during the COVID-19 pandemic: a cross-sectional study. Psychiatry Res. 2020;288:112936. doi:10.1016/j.psychres.2020.112936

43. Aboalshamat K, Hou XY, Strodl E. Psychological well-being status among medical and dental students in Makkah, Saudi Arabia: a cross-sectional study. Med Teach. 2015;37(Suppl 1):S75-81. doi:10.3109/0142159X.2015.1006612

44. Ahmad FA, Karimi AA, Alboloushi NA, Al-Omari QD, AlSairafi FJ, Qudeimat MA. Stress level of dental and medical students: comparison of effects of a subject-based curriculum versus a case-based integrated curriculum. $J$ Dent Educ. 2017;81(5):534-544. doi:10.21815/JDE.016.026

45. Murphy RJ, Gray SA, Sterling G, Reeves K, DuCette J. A comparative study of professional student stress. J Dent Educ. 2009;73(3):328-337. doi:10.1002/j.0022-0337.2009.73.3.tb04705.x

46. Ahmed MA, Jouhar R, Ahmed N, et al. Fear and practice modifications among dentists to combat novel coronavirus disease (COVID-19) outbreak. Int J Environ Res Public Health. 2020;17 (8):2821. doi:10.3390/ijerph17082821

47. Sarfaraz S, Shabbir J, Mudasser MA, et al. Knowledge and attitude of dental practitioners related to disinfection during the COVID-19 pandemic. Healthcare (Basel, Switzerland). 2020;8(3).

48. Roberto A, Almeida A. [Mental health of students of medicine: exploratory study in the Universidade da Beira Interior]. Acta Med Port. 2011;24(Suppl 2):279-286.

49. Abouammoh N, Irfan F, AlFaris E. Stress coping strategies among medical students and trainees in Saudi Arabia: a qualitative study. BMC Med Educ. 2020;20(1):124. doi:10.1186/s12909-020-02039-y

50. Geldsetzer P. Use of rapid online surveys to assess people's perceptions during infectious disease outbreaks: a cross-sectional survey on COVID-19. J Med Internet Res. 2020;22(4):e18790. doi:10.2196/ 18790
Risk Management and Healthcare Policy

\section{Publish your work in this journal}

Risk Management and Healthcare Policy is an international, peerreviewed, open access journal focusing on all aspects of public health, policy, and preventative measures to promote good health and improve morbidity and mortality in the population. The journal welcomes submitted papers covering original research, basic science, clinical \& epidemiological studies, reviews and evaluations, guidelines, expert opinion and commentary, case reports and extended reports. The manuscript management system is completely online and includes a very quick and fair peer-review system, which is all easy to use. Visit http://www.dovepress.com/testimonials.php to read real quotes from published authors. 